\title{
Random Illumination Microscopy from Variance Images
}

\author{
$1^{\text {st }}$ Simon Labouesse \\ Aix Marseille Université, CNRS, IBDM-UMR7288 \\ Turing Center for Living Systems, Marseille, France \\ simon.labouesse@colorado.edu
}

\author{
$2^{\text {nd }}$ Jérôme Idier \\ Laboratoire des Sciences du Numérique de Nantes, CNRS \\ F-44321 Nantes, France \\ jerome.idier@1s2n.fr
}

\author{
$3^{\text {rd }}$ Anne Sentenac \\ Institut Fresnel, CNRS \\ F-13397 Marseille, France \\ anne.sentenac@fresnel.fr
}

\author{
$4^{\text {th }}$ Thomas Mangeat \\ Centre de Biologie Intégrative, CNRS \\ F-31062 Toulouse, France \\ thomas.mangeat@univ-tlse3.fr
}

\author{
$5^{\text {th }}$ Marc Allain \\ Institut Fresnel, CNRS \\ F-13397 Marseille, France \\ marc.allain@fresnel.fr
}

\begin{abstract}
We propose a reconstruction algorithm called algoRIM for super-resolution fluorescence microscopy, based on speckle illuminations and image variance matching. Superresolution with a factor two or close can be achieved under realistic conditions in terms of number of images and signal to noise ratio. Here, our key result is an approximation of the statistical variance equation, leading to a drastic reduction of the computational complexity. Moreover, we demonstrate that the unmodulated out-of-focus light does not contribute to the data variance, and that the statistical component due to noise can be estimated and removed in an unsupervised way, which is a crucial contribution to the practical robustness of algoRIM.

Index Terms-Fluorescence microscopy, structured illumina-
\end{abstract} tion, super-resolution, optical sectioning, variance, estimation

\section{INTRODUCTION}

Fluorescence widefield microscopy is a decisive imaging tool for biology. However, the spatial resolution is limited by the diffraction barrier, around $300 \mathrm{~nm}$. Imaging subcellular structures requires to improve the resolution beyond this barrier. Furthermore, phototoxicity limits the number of photons that can be sent to the sample. In such conditions, structured illumination microscopy (SIM) reaches an excellent trade-off between spatial and temporal resolution, and low toxicity [1][3]. SIM consists in recording several low-resolution images of the sample illuminated with known periodic illuminations with different positions and orientations. The periodic illumination of SIM permits to downmodulate the previously inaccessible high spatial frequency components of the sample inside the low-resolution images. The super-resolved reconstruction is obtained through a joint demodulation process using the illumination as a carrier wave. Such a data processing step requires the precise knowledge of the illumination pattern, thus a tight control of the latter, which makes SIM difficult to use

The authors acknowledge partial financial support for this paper from the Agence Nationale de la Recherche under Grant ANR-12-BS03-0006 and the ITMO Cancer of the 'Plan Cancer 2014-2019'.

This work was carried out in part while $\mathrm{S}$. Labouesse was a $\mathrm{PhD}$ student at Institut Fresnel, Marseille, France, and later in post-doctoral position at the University of Colorado Boulder, USA. and limits its application to weakly scattering samples [4]. SIM is prone to introduce artifacts in the reconstruction as soon as the illuminations are distorted at the sample plane, because of imperfect calibration, or because of the diffusion in the thickness of the sample [1], [5].

Random illumination microscopy (RIM) was proposed as a more robust imaging technique than SIM [6]. RIM consists in shining the sample with several uncontrolled speckles and in extracting a super-resolved image from the multiple low frequency images acquired. The maximum super-resolution gain of RIM is the same as SIM [7], i.e., a factor of two in the lateral dimensions. On the other hand, RIM requires only simple modifications of a fluorescent microscope, and it is easy to use [8]. Moreover, it is robust to optical aberrations, which makes it an excellent candidate for functional cell imaging. Some RIM algorithms jointly estimate the object and the illuminations using positivity and/or sparsity constraints [9], [10]. However, the latter algorithms do not provide a guaranteed super-resolution gain, and they are not robust to the presence of an umodulated background. Moreover, their computational complexity (CC) is proportional to the number of acquired images.

An alternative is to estimate the fluorescence map marginally on the unknown illuminations, using a method of moments based on matching some statistics of the acquired images. Such a method is asymptotically consistent [11], and it does not rely on a prior knowledge about the quantity of interest. In [7], a Kullback Leibler divergence is used as a contrast function between the empirical and statistical second order moments. However, this estimator relies on image covariance matching, which has a prohibitive cost of $\mathcal{O}\left(N^{2}\right)$ and $\mathcal{O}\left(N^{3}\right)$ in terms of memory and CC for $N$-pixel images, respectively. A patch method was investigated with a reduction of the $\mathrm{CC}$ to $\mathcal{O}\left(N^{2} \log N\right)$ [12].

In this paper, we propose a new reconstruction algorithm called algoRIM, based on variance matching. We introduce an approximation of the statistical variance equation leading to a $\mathrm{CC}$ of $\mathcal{O}(N \log N)$.We also show that it is possible to 
separate the statistical contribution of the "useful" photons from the unwanted components, in a fully unsupervised way. The latter components include both the electronic and the photon counting noise, but also the fluorescence light emitted from out-of-focus planes, real tissues being three-dimensional. AlgoRIM was recently applied to cases of super-resolved functional imaging of living cells and tissues [8], which confirms its practical super-resolution capability, as well as its versatility and robustness.

In Section II, we introduce the discretized model for RIM and we express second-order statistical moments of the measurements which are useful in the rest of the paper. In Section III, a reformulation of the statistical variance is proposed, which is a key part of algoRIM, leading to a substantial reduction of the computational complexity. Section IV proves that the noise component of the data variance can be estimated from the acquired images. In Section V, we give some details about the adopted objective function and minimization strategy. In Section VI, simulation results illustrate the capacity of algoRIM to estimate a super-resolved image of the object in presence of noise and of an unmodulated background. We also provide a two-color fluorescence super-resolved reconstruction from experimental RIM images of podosomes.

\section{Model And Statistical Moments}

Let us first derive the statistical variance of the RIM images. We restrict ourselves to the problem in a $2 \mathrm{D}$ fully discrete setting, where both the recorded images and the sample are represented on regular grids, with a sampling rate common to both, and we assume a linear invariant fluorescence microscope with a linear response of the fluorophores. Then, the $m$ th noiseless acquired image reads

$$
\boldsymbol{x}_{m}=\boldsymbol{H D i a g}\left(\boldsymbol{I}_{m}\right) \boldsymbol{\rho}+\boldsymbol{b}
$$

where

- $\boldsymbol{H}$ is the convolution matrix corresponding to the point spread function (PSF) of the microscope. Here, $\boldsymbol{H}$ is assumed known, real symmetric, and block circulant with circulant blocks (BCCB), and we call optical transfer function (OTF) the Fourier transform of the PSF.

- $\boldsymbol{I}_{m}$ the $m$ th unknown speckle illumination,

- $\rho$ the unknown fluorophore concentration, which is the only quantity of interest.

- $\boldsymbol{b}$ is an unknown background corresponding to the contribution of out-of-focus planes. Here, it is considered as a deterministic, unmodulated component.

Finally, Diag() defines a diagonal matrix from a vector. Without loss of generality, the spatial sampling rate is normalized to unity in each direction. Since our goal is to demonstrate a factor two in terms of super-resolution, the normalized cutoff frequency $f_{\text {cut }}$ imposed by the PSF is assumed to be smaller than 1/4. Experimental setups are usually designed so that $f_{\text {cut }}$ is close to $1 / 2$, but for the sake of simplicity, we do not include the numerical oversampling required in our model.
We assume that a spatially uncorrelated noise pledges the measurements, so that the acquired images read

$$
\boldsymbol{y}_{m} \sim \mathcal{P}\left(\boldsymbol{y}_{m} \mid \boldsymbol{x}_{m}\right),
$$

where $\mathcal{P}$ is the conditional distribution of $\boldsymbol{y}_{m}$ given $\boldsymbol{x}_{m}$, induced by the presence of noise. In a typical way, both the electronic noise and the photon counting noise can be taken into account simultaneously using (2). We postulate the statistical expectation of the image $\boldsymbol{y}_{m}$ knowing the illumination $\boldsymbol{I}_{m}$ to be equal to the noiseless measurement, i.e., $\mathcal{E}\left(\boldsymbol{y}_{m} \mid \boldsymbol{I}\right)=\boldsymbol{x}_{m}$. We also assume that the illuminations are fully developed speckles with known stationary second order statistics:

$$
\begin{aligned}
\boldsymbol{\mu}_{\boldsymbol{I}} & =\mathcal{E}\left(\boldsymbol{I}_{m}\right) \\
\boldsymbol{C}=\boldsymbol{\Sigma}_{\boldsymbol{I}} & =\mathcal{E}\left(\left(\boldsymbol{I}_{m}-\boldsymbol{\mu}_{\boldsymbol{I}}\right)\left(\boldsymbol{I}_{m}-\boldsymbol{\mu}_{\boldsymbol{I}}\right)^{\mathrm{t}}\right),
\end{aligned}
$$

$\boldsymbol{C}$ being a real symmetric BCCB matrix.

The spatial correlation of the acquired images can be expressed using the law of total covariance:

$$
\Sigma_{y}=\Sigma_{x}+\operatorname{Diag}(w)
$$

where

$$
\Sigma_{x}=H \operatorname{Diag}(\rho) C \operatorname{Diag}(\rho) H
$$

is the covariance of the expectation of $\boldsymbol{y}$ given $\boldsymbol{I}$, and $\operatorname{Diag}(\boldsymbol{w})$ is the covariance of the noise component, $\boldsymbol{w}$ being an unknown map of noise variance. Let us stress here that $\boldsymbol{\Sigma}_{\boldsymbol{x}}$ does not depend on the background component $\boldsymbol{b}$, in contrast with the first-order statistics

$$
\mu_{y}=\mu_{x}=H \operatorname{Diag}(\rho) \mu_{I}+b .
$$

Such a property corresponds to the sectioning capacity of speckle illumination microscopy, as already noticed in [13], [14]. Finally, the statistical variance of $\boldsymbol{y}$ is simply the diagonal part of $\boldsymbol{\Sigma}_{y}$ :

$$
v_{y}=\operatorname{diag}\left(\Sigma_{y}\right)=v_{x}+w
$$

with $\boldsymbol{v}_{\boldsymbol{x}}=\operatorname{diag}\left(\boldsymbol{\Sigma}_{x}\right)$. We temporarily assume $\boldsymbol{w}$ to be known, Section IV being focused on an estimation procedure for $\boldsymbol{w}$. Unfortunately, computing $\boldsymbol{\Sigma}_{\boldsymbol{x}}$ using (3) costs $\mathcal{O}\left(N^{3}\right)$, as well as computing $\boldsymbol{v}_{\boldsymbol{x}}$ alone. However, the computation of $\boldsymbol{v}_{\boldsymbol{x}}$ can be reformulated, leading to an efficient approximation scheme, as shown below.

\section{STATISTICAL VARIANCE REFORMULATION}

Each element of $\boldsymbol{v}_{\boldsymbol{x}}$ has a quadratic expression with respect to the object: $\left[\boldsymbol{v}_{\boldsymbol{x}}\right]_{i}=\boldsymbol{\rho}^{\mathrm{t}} \boldsymbol{T}^{(i)} \boldsymbol{\rho}$, with

$$
\boldsymbol{T}^{(i)}=\operatorname{Diag}\left([\boldsymbol{H}]_{i,:}\right) \boldsymbol{C} \operatorname{Diag}\left([\boldsymbol{H}]_{i,:}\right),
$$

where $[\boldsymbol{H}]_{i,:}$ is the $i$ th row of $\boldsymbol{H}$. Matrices $\boldsymbol{T}^{(i)}$ are real nonnegative definite, so they admit an eigendecomposition. Therefore,

$$
\left[\boldsymbol{v}_{\boldsymbol{x}}\right]_{i}=\sum_{k=1}^{N}\left(\boldsymbol{\rho} \circ \boldsymbol{m}^{(k, i)}\right)^{\mathrm{t}}\left(\boldsymbol{\rho} \circ \boldsymbol{m}^{(k, i)}\right)
$$




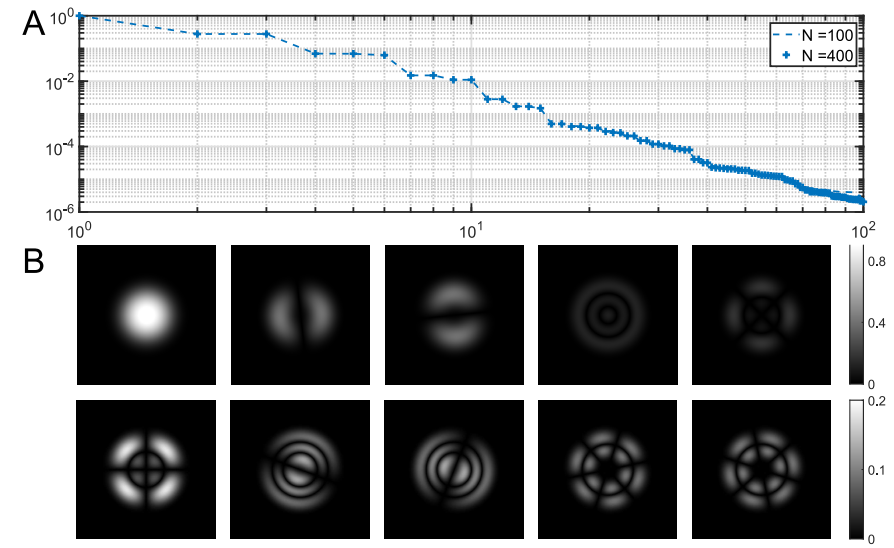

Fig. 1. Eigenvalues and vectors of matrices $\boldsymbol{T}^{(i)}$. A) Eigenvalues of $\boldsymbol{T}^{(0)}$ for $N=100$ and $N=400$ normalized by the first eigenvalue. B) From top left to bottom right, the amplitude of the Fourier transform of the eigenvectors $\left|\widetilde{\boldsymbol{m}}^{(k, i)}\right|$ for $N=400$.

with $\boldsymbol{m}^{(k, i)}=\sqrt{s^{(k, i)}} \boldsymbol{u}^{(k, i)}$, where $s^{(k, i)} \geqslant 0$ and $\boldsymbol{u}^{(k, i)}$ are the $k$ th eigenvalue and eigenvector of $\boldsymbol{T}^{(i)}$, respectively, and $\circ$ is the Hadamard (i.e., entrywise, or Schur) matrix product [15, Chap.5]. One can notice that matrices $\boldsymbol{T}^{(i)}$ are related one to each other by two similar permutations along both the rows and columns (see Eq. (4)). Therefore, they share the same spectrum and the same rank $R \leqslant N$, and their eigenvectors are related one to each other through the same permutation [16]. Any element $k$ of the sum in (5) can be computed for all pixels $i$ simultaneously by convolution with a filter $\boldsymbol{m}^{(k, 0)}$, due to the BCCB structure of $\boldsymbol{H}$, and therefore

$$
\boldsymbol{v}_{\boldsymbol{x}}=\sum_{k=1}^{K}\left|\boldsymbol{M}^{(k)} \boldsymbol{\rho}\right|^{2}
$$

with $K=N$ and $\boldsymbol{M}^{(k)}=\mathbf{B C C B}\left(\boldsymbol{m}^{(k, 0)}\right), \mathbf{B C C B}()$ being the BCCB matrix with the input vector as its first row. Exact computations are obtained if $K \geqslant R$ (let us remark that matrices $\boldsymbol{T}^{(i)}$ are not full rank). In Fig. 1A, the decrease speed of the eigenvalues $s^{(k, 0)}$ are shown for $N=100$ and $N=400$. The decrease is very fast, and almost identical for the two cases. Hereafter, only $K=10$ terms will be kept with the corresponding 2D Fourier transform of the $m^{(k, 0)}$ shown in Fig. 1B.

\section{CONDITIONAL NOISE VARIANCE ESTIMATION}

In this section, we demonstrate that the conditional variance $\boldsymbol{w}$ can be estimated from an asymptotically large number of images, and we deduce a practical estimator.

Theorem 1. If RIM images are sampled above the Nyquist-Shannon sampling limit, then the conditional variance $\boldsymbol{w}$ is identifiable from an asymptotically large number of images $\boldsymbol{y}_{m}$.

\section{A. Proof of Theorem 1}

We call $\mathcal{D}_{\mathrm{PSF}}$ the support of the OTF, we also define $\widetilde{\boldsymbol{a}}=$ $\mathbf{1}_{\overline{\mathrm{PSF}}}$ as the indicator function of the complement of $\mathcal{D}_{\mathrm{PSF}}$ :

$$
[\widetilde{\boldsymbol{a}}]_{i}= \begin{cases}0 & \text { for all } i \text { inside the OTF domain, } \\ 1 & \text { otherwise }\end{cases}
$$

$\boldsymbol{A}=\mathbf{B C C B}(\boldsymbol{a})$ is the convolution matrix by the $2 \mathrm{D}$ inverse Fourier transform of $\widetilde{\boldsymbol{a}}$. By definition, we have $\boldsymbol{A} \boldsymbol{H}=\boldsymbol{H} \boldsymbol{A}=$ 0. Let us define the intermediate variables $\boldsymbol{z}_{m}=\boldsymbol{A} \boldsymbol{y}_{m}$. Their covariance matrix is $\boldsymbol{\Sigma}_{\boldsymbol{z}}=\boldsymbol{A} \boldsymbol{\Sigma}_{\boldsymbol{y}} \boldsymbol{A}^{\mathrm{t}}=\boldsymbol{A D i a g}(\boldsymbol{w}) \boldsymbol{A}^{\mathrm{t}}$, and their statistical variance is [17]

$$
\boldsymbol{v}_{\boldsymbol{z}}=\operatorname{diag}\left(\boldsymbol{A D i a g}(\boldsymbol{w}) A^{\mathrm{t}}\right)=(\boldsymbol{A} \circ \boldsymbol{A}) \boldsymbol{w} .
$$

Moreover, as the number $M$ of images grows, the sample variance of $\left\{\boldsymbol{z}_{m}\right\}_{m=1 \ldots M}$ converges towards $\boldsymbol{v}_{z}$.

Proposition 1. If RIM images are sampled above the Nyquist-Shannon sampling limit, then $\boldsymbol{A} \circ \boldsymbol{A}$ is an invertible matrix.

Proof: Matrix $\boldsymbol{A} \circ \boldsymbol{A}$ is a convolution matrix with the filter $|\boldsymbol{a}|^{2}$. The 2D inverse Fourier transform of $|\boldsymbol{a}|^{2}$ is $\widetilde{\boldsymbol{a}} \otimes \widetilde{\boldsymbol{a}}$ with $\otimes$ the convolution operator. The circular convolution operation is

$$
[\widetilde{\boldsymbol{a}} \otimes \widetilde{\boldsymbol{a}}]_{i, j}=\sum_{m, n}[\widetilde{\boldsymbol{a}}]_{m, n}[\widetilde{\boldsymbol{a}}]_{i-m(\bmod N), j-n(\bmod N)}
$$

where vectors are indexed by two indices going from 0 to $N-1$ to identify individual elements of the two-dimensional normalized frequency grid (NFG). We remark that $[\widetilde{\boldsymbol{a}}]_{i, 0}=$ $1, \forall i$ and $[\widetilde{\boldsymbol{a}}]_{0, j}=1, \forall j$ because such components correspond to positions at the boundary of the NFG, and the sampling rate is above the Nyquist-Shannon sampling limit. Elements of the sum (7) are either 0 or 1 . However, particular terms of the sum can be shown to be 1: indeed, $[\widetilde{\boldsymbol{a}}]_{0, j}[\widetilde{\boldsymbol{a}}]_{i, 0}=1, \forall(i, j)$, and therefore $[\widetilde{\boldsymbol{a}} \otimes \widetilde{\boldsymbol{a}}]_{i, j}>0, \forall(i, j)$. Matrix $\boldsymbol{A} \circ \boldsymbol{A}$ is thus positive definite and therefore invertible.

As an estimator of the conditional variance, we propose $\widehat{\boldsymbol{w}}=(\boldsymbol{A} \circ \boldsymbol{A})^{-1} \widetilde{\boldsymbol{v}}_{\boldsymbol{z}}$ with $\widetilde{\boldsymbol{v}}_{\boldsymbol{z}}$ the empirical variance of images $\boldsymbol{z}_{m}$. This estimation step is especially useful because of the Poisson noise component, which makes the conditional noise variance depend on the unknown map $\rho$, but also on the unknown background light $\boldsymbol{b}$.

\section{Algorithm}

In order to estimate the object $\rho$, we rely on the matching of the variance,

$$
F(\boldsymbol{\rho})=D\left(\widehat{\boldsymbol{v}}_{y}-\widehat{\boldsymbol{w}}-\boldsymbol{v}_{\boldsymbol{x}}(\boldsymbol{\rho})\right),
$$

using a simple least-square fit $D(\cdot)=\|\cdot\|_{2}^{2}$. We perform local descent on $F$ using an L-BFGS scheme [18] with a memory size of 10 . The optimal step size is calculated by solving a single variable polynomial of degree three at each iteration. The gradient of $F$ is given by

$$
\frac{\partial F(\boldsymbol{\rho})}{\partial \boldsymbol{\rho}}=4 \sum_{k=1}^{K} \boldsymbol{M}^{(k)^{\mathrm{t}}}\left(\left(\boldsymbol{M}^{(k)} \boldsymbol{\rho}\right) \circ \boldsymbol{r}(\boldsymbol{\rho})\right)
$$



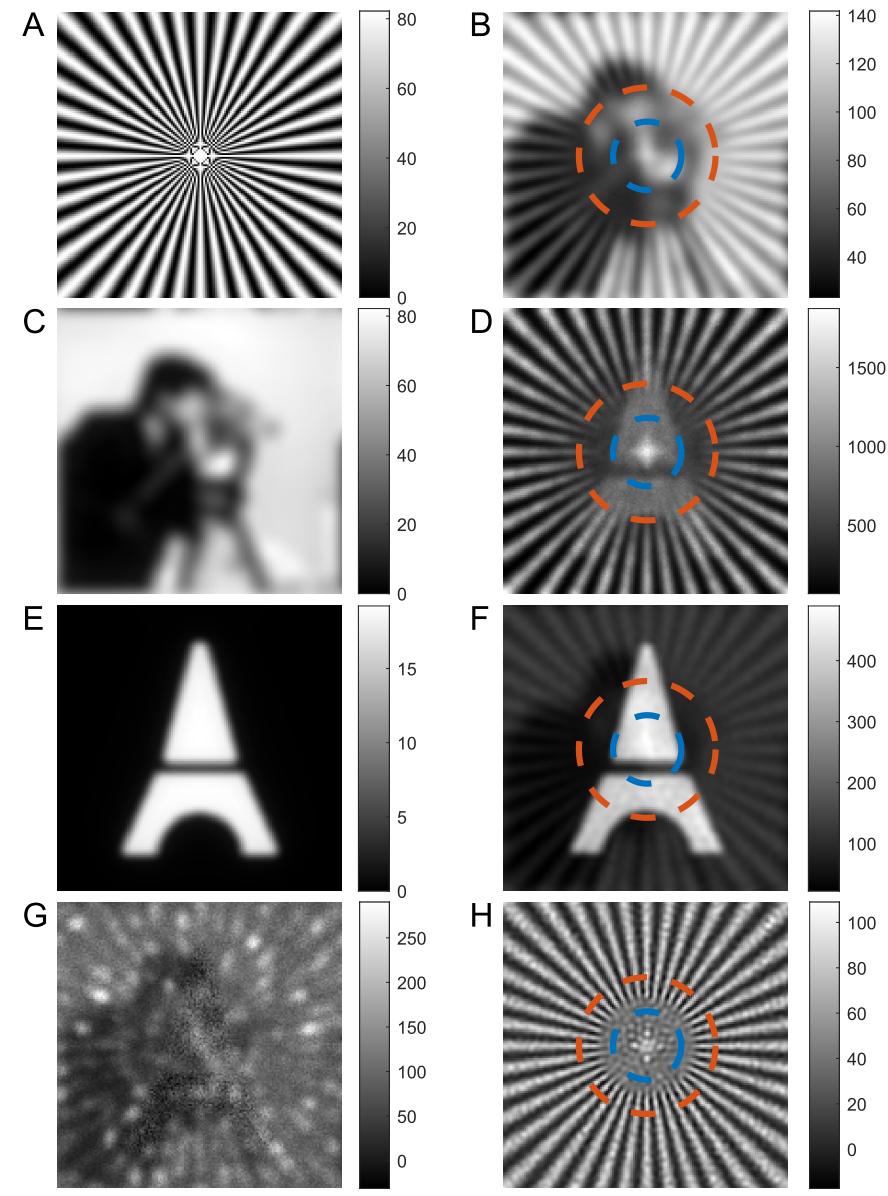

Fig. 2. Simulation results from 1000 noisy RIM images. A) Siemens target object $\rho$, B) mean of the RIM images $\boldsymbol{\mu}_{\boldsymbol{y}}$, C) unmodulated background $\boldsymbol{b}$, D) variance of the RIM images $\boldsymbol{v}_{\boldsymbol{y}}$, E) standard deviation of additive Gaussian noise, F) estimated conditional variance $\boldsymbol{w}, G)$ one noisy RIM image, $\mathrm{H}$ ) reconstruction output from algoRIM. Red and blue circles indicate the limits of the regions that are non-resolvable with uniform and structured illuminations, respectively.

with $\boldsymbol{r}(\boldsymbol{\rho})=\widehat{\boldsymbol{v}}_{y}-\widehat{\boldsymbol{w}}-\boldsymbol{v}_{\boldsymbol{x}}(\boldsymbol{\rho})$. Let us remark that the least square fit may be replaced by a more accurate measure with a better statistical justification. Moreover, in practice, a preliminary Wiener deconvolution step is performed on the RIM images. This step produces a whitening effect, which improves the statistical variance approximation, more information being concentrated in the variance component.

\section{RESULTS}

To illustrate the performances of algoRIM, we simulate 1000 RIM images like the one in Fig. 2G. We assume $\boldsymbol{C} \propto \boldsymbol{H}$, which is roughly the case in epi-fluoresence if we neglect the Stokes shift. We use a Siemens star object (see Fig. 2A) and speckle illuminations. A filtered version of the cameraman image is added as an unmodulated background $\boldsymbol{b}$. Let us remark that it is reasonable to assume that $\boldsymbol{b}$ has no spatial frequency content outside the support of the OTF. Images are scaled by a multiplicative factor so that after the photon counting noise is simulated, the overall signal to noise ratio is

$$
\mathrm{SNR}=10 \log \left(\frac{\sum_{m, i}\left[\boldsymbol{x}_{m}\right]_{i}^{2}}{\sum_{m, i}\left[\boldsymbol{y}_{m}-\boldsymbol{x}_{m}\right]_{i}^{2}}\right)
$$

is $50 \mathrm{~dB}$. Then an additive Gaussian noise with a standard deviation displayed in Fig. 2E is added. The mean of all RIM images is shown in Fig. 2B. A red circle indicates the limit of the OTF support, the inner region being nonresolvable in the widefield image. Moreover, the background artefact is clearly visible and could lead to misinterpretations in real widefield fluorescence microscopy. The variance of all RIM images is shown in Fig. 2D. It contains smaller details inside the red circle. However, $\boldsymbol{v}_{\boldsymbol{x}}$ is not linearly related to the object (see Eq. (6)) and the presence of the Gaussian noise is visible. Relative intensities are not preserved, so no quantitative evaluations can be retrieved in a direct way from $\widehat{\boldsymbol{v}}_{\boldsymbol{x}}$ nor $\sqrt{\widehat{\boldsymbol{v}}_{\boldsymbol{x}}}$.

In Fig. $2 \mathrm{~F}$, the estimated conditional variance $\widehat{\boldsymbol{w}}$ is shown. Finally, Fig. $2 \mathrm{H}$ shows the reconstructed object $\widehat{\rho}$ using algoRIM. The blue circle indicates the region of the Siemens target that is non-resolvable, even using structured illumination. We can see that the object is reconstructed almost up to the blue circle, and much better than what is directly visible in the variance image. In simulations with more than 10000 RIM images and no noise, the reconstructed images perfectly reach the blue circle.

In Fig. 3, we show results obtained from real data acquired with a two-color fluorescence microscope described in [8]. The sample is a cell membrane with marked podosomes (lifeact-GFP in red and zyxine-mCh in green). Podosomes are composed of an approximately $500 \mathrm{~nm}$ high and large F-actin protrusive core surrounded by an adhesion ring. In Fig. 3, we see a resolution improvement in the reconstructed image compared to the deconvolved widefield image. In the deconvolved widefield image, we observe an undesired low frequency hallow, probably due to out-of-focus light, which is perfectly rejected in the reconstructed image.
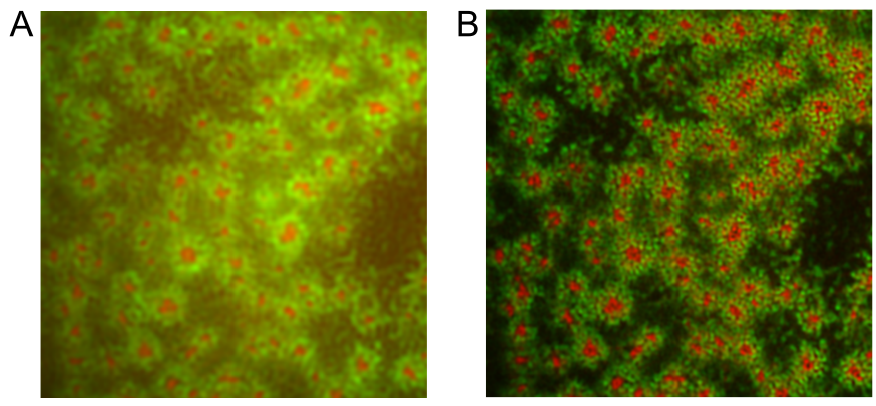

Fig. 3. Real data reconstruction of podosomes from 800 illuminations and an exposition time of $10 \mathrm{~ms}$ per image. A) Widefield image simulated by taking the average of all the RIM images, B) Reconstruction output of algoRIM.

\section{CONCLUSION}

AlgoRIM is a fast algorithm dedicated to RIM, based on the minimization of a distance between the statistical 
variance and the empirical variance of the acquired images. An approximation computation of the statistical variance leads to a reduction of the complexity to $\mathcal{O}(N \log N)$. We have proposed an estimator of the conditional noise statistical variance suited to noise distributions encountered in microscopy. Simulations clearly show the capacity of algoRIM to recover a super resolved image of the object even in presence of an unmodulated background and of photon noise. We have also illustrated the super-resolution capacity of algoRIM, as well as the rejection of out of focus light on a real data case.

\section{REFERENCES}

[1] Y. Wu and H. Shroff, "Faster, sharper, and deeper: structured illumination microscopy for biological imaging," Nature methods, vol. 15, no. 12, pp. 1011-1019, 2018.

[2] M. G. Gustafsson, "Surpassing the lateral resolution limit by a factor of two using structured illumination microscopy," Journal of microscopy, vol. 198 , no. 2, pp. 82-87, 2000.

[3] R. Heintzmann and C. G. Cremer, "Laterally modulated excitation microscopy: improvement of resolution by using a diffraction grating," in Optical Biopsies and Microscopic Techniques III, vol. 3568. International Society for Optics and Photonics, 1999, pp. 185-196.

[4] J. Demmerle, C. Innocent, A. J. North, G. Ball, M. Müller, E. Miron, A. Matsuda, I. M. Dobbie, Y. Markaki, and L. Schermelleh, "Strategic and practical guidelines for successful structured illumination microscopy," Nature protocols, vol. 12, no. 5, p. 988, 2017.

[5] R. Ayuk, H. Giovannini, A. Jost, E. Mudry, J. Girard, T. Mangeat, N. Sandeau, R. Heintzmann, K. Wicker, K. Belkebir et al., "Structured illumination fluorescence microscopy with distorted excitations using a filtered blind-SIM algorithm," Optics letters, vol. 38, no. 22, pp. 47234726, 2013.

[6] E. Mudry, K. Belkebir, J. Girard, J. Savatier, E. Le Moal, C. Nicoletti, M. Allain, and A. Sentenac, "Structured illumination microscopy using unknown speckle patterns," Nature Photonics, vol. 6, no. 5, pp. 312-315, 2012.

[7] J. Idier, S. Labouesse, M. Allain, P. Liu, S. Bourguignon, and A. Sentenac, "On the superresolution capacity of imagers using unknown speckle illuminations," IEEE Transactions on Computational Imaging, vol. 4, no. 1, pp. 87-98, 2018.

[8] T. Mangeat, S. Labouesse, M. Allain, E. Martin, R. Poincloux, A. Bouissou, S. Cantaloube, E. Courtaux, E. Vega, T. Li et al., "Super-resolved live-cell imaging using random illumination microscopy," bioRxiv, 2020.

[9] S. Labouesse, A. Negash, J. Idier, S. Bourguignon, T. Mangeat, P. Liu, A. Sentenac, and M. Allain, "Joint reconstruction strategy for structured illumination microscopy with unknown illuminations," IEEE Transactions on Image Processing, vol. 26, no. 5, pp. 2480-2493, 2017.

[10] L.-H. Yeh, L. Tian, and L. Waller, "Structured illumination microscopy with unknown patterns and a statistical prior," Biomedical optics express, vol. 8, no. 2, pp. 695-711, 2017.

[11] A. W. Van der Vaart, Asymptotic statistics. Cambridge University Press, 2000, vol. 3.

[12] P. Liu, J. Idier, S. Bourguignon, S. Labouesse, M. Allain, and A. Sentenac, "Minimum contrast estimation for super-resolution fluorescence microscopy using speckle patterns," in $26^{e}$ coll. GRETSI, Juan-les-Pins, France, Sep. 2017.

[13] C. Ventalon and J. Mertz, "Quasi-confocal fluorescence sectioning with dynamic speckle illumination," Optics letters, vol. 30, no. 24, pp. 3350$3352,2005$.

[14] D. Lim, K. K. Chu, and J. Mertz, "Wide-field fluorescence sectioning with hybrid speckle and uniform-illumination microscopy," Optics letters, vol. 33, no. 16, pp. 1819-1821, 2008.

[15] R. Horn and C. Johnson, Matrix analysis. Cambridge University Press, 1991.

[16] A. Quarteroni, R. Sacco, and F. Saleri, Numerical mathematics. Springer Science \& Business Media, 2010, vol. 37.

[17] K. B. Petersen and M. S. Pedersen, The Matrix Cookbook. Technical University of Denmark, Nov. 2012.

[18] D. C. Liu and J. Nocedal, "On the limited memory BFGS method for large scale optimization," Mathematical programming, vol. 45, no. 1-3, pp. 503-528, 1989. 